\title{
MODELLING OF A DETERMINISTIC EMULATOR TO ACCURATELY REPRODUCE THE REAL FIBER AFFECTED BY PMD UP TO THIRD ORDER
}

\author{
Antonella Bogoni ${ }^{1}$, Alessandra Orlandini ${ }^{2}$, Luca Poti ${ }^{3}$ \\ ${ }^{1}$ Consorzio Nazionale Interuniversitario per le Telecomunicazioni (CNIT), Via Matteucci 34/l, \\ 56124 Pisa-Italy \\ ${ }^{2}$ Università degli Studi di Parma, Dipartimento di Ingegneria dell'Informazione, Parco Area \\ delle Scienze 181/A, 43100 Parma - Italy \\ ${ }^{3}$ Consorzio Nazionale Interuniversitario per le Telecomunicazioni (CNIT), Via Matteucci $34 /$, \\ 56124 Pisa-Italy
}

Abstract: A novel deterministic PMD emulator, consisting of delay-sections coupled by rotators, is proposed. The stochastic behavior of its parameters is analysed. By opportunely choosing the rotation angles, this emulator is able to reproduce the PMD statistics up to third order with a reduced number of sections.

Key words: Optical fiber, optical communication systems, polarization mode dispersion (PMD), PMD emulator.

\section{INTRODUCTION}

In a first order approximation, polarization mode dispersion (PMD), that is described by a differential group delay (DGD) between two orthogonal states of polarization (principal states of polarization or PSPS), causes an undesired output pulse broadening; the frequency dependence of DGD and PSPs generates other distorting effects, considered as higher order effects. Moreover, because of its random variations, the phenomenon has to be statistically studied.

Nowadays PMD can be considered as one of the major source of limitation to the performance of long distance high-bit rate optical systems. The possibility of truthfully recreating the real PMD effects of an optical link is extremely useful for a proper design of a high-performance system. 
As regards this point, an effective tool is represented by the PMD emulator, that also conveniently serves the purpose of testing the PMD compensators capability. This device is designed to describe the optical fiber as the concatenation of several birefringent elements with fixed DGD (such as polarization maintaining fibers [2] or birefringent crystals [1], [3]) properly coupled by polarization controllers or rotators [1]-[4]. The number of the delay sections used and the choice of the coupling conditions are essential for the accurate representation of PMD stochastic behavior [2]-[4].

In this paper we present a deterministic PMD emulator with delay sections coupled by rotators. The proposed device is called "deterministic" because it can emulate for a particular given PMD condition (we fix that up to the second order).

By varying the rotation angles, a refined map of correspondence between them and the PMD parameters introduced by the emulator is found. The requested PMD condition can be obtained by extracting the necessary correspondent coupling angles from the above-mentioned map.

As shown in literature, a statistical reproduction of a real fiber is generally obtained by choosing the rotation angles with a uniform probability density function (PDF). To correctly describe the Maxwellian PDF of DGD, at least 15 sections are needed [2]. In the present work, we show that, by an opportune choice of the coupling angles statistics, this number of sections can be reduced and it is also sufficient to well represent high-order PMD statistics. In fact, by using a small number of delay sections, our emulator reproduces the statistical behavior of a real PMD fiber with a great accuracy.

For varying PMD conditions, the emulated probability density functions calculated up to second order are compared to their known analytical expressions [6], [7], showing a good agreement even on the tails. The results of third order achieve a good approximation of the ones obtained with the Discrete Random Waveplate (DRW) numerical model, and they satisfy the analytical relationship between the mean square value of second- and zero-order derivative of the dispersion vector [8].

\section{MULTI-SECTION PMD EMULATOR: THEORY}

The emulator presented in this work consists of $\mathrm{N}\left(\mathrm{N}_{\min }=3, \mathrm{~N}_{\min }=8\right)$ sections with fixed DGD $\delta \tau_{\mathrm{i}}$ and $\mathrm{N}-1$ rotators with coupling angles $\theta_{i}$, which can be varied with different steps. If the Jones matrix of each section is 


$$
U_{i}=\left[\begin{array}{cc}
\cos \theta_{i} & -\sin \theta_{i} \\
\sin \theta_{i} & \cos \theta_{i}
\end{array}\right] \cdot\left[\begin{array}{cc}
e^{\frac{j \delta \tau_{i} \omega}{2}} & 0 \\
0 & e^{-\frac{j \delta \tau_{i} \omega}{2}}
\end{array}\right],
$$

the Jones matrix of the whole emulator is

$$
U=\prod_{0}^{N-1} U_{N-i}=\left[\begin{array}{cc}
u_{1} & u_{2} \\
-U_{2}^{*} & u_{1}^{*}
\end{array}\right] \quad i=1, \ldots, N
$$

The original idea provides, by using an opportune software, for an exhaustive scan for all rotation angles $\theta_{i}$, so that a map of correspondence between them and the PMD emulator parameters can be created. By this way, it is possible choosing any given combination of PMD parameters and extracting the correspondent right angles to be set at the emulator. If the analytically known PMD statistics [6], [7] are imposed to the emulator, the correspondent statistics for the coupling angles can be found. In the following section it will be shown that the precision given by the statistics of the emulated parameters depends on $\mathrm{N}$ and the step-angle accuracy.

Now, in order to describe the statistical behavior of the high-order PMD parameters, it is worth introducing the three-dimensional frequencydependent dispersion vector $\vec{\Omega}$ that characterizes the PMD of an optical fiber in the Stokes space. For a fixed $\omega$, its module is equal to the fiber DGD and it has the direction of the slow PSP. In particular, a Taylor expansion of order $n-1$ at center-bandwidth of $\vec{\Omega}$

$$
\vec{\Omega}=\vec{\Omega}_{0}+\vec{\Omega}_{0}^{\prime}\left(\omega-\omega_{0}\right)+\ldots+\vec{\Omega}_{0}^{(n-1)} \frac{\left(\omega-\omega_{0}\right)^{(n-1)}}{(n-1) !}
$$

represents a PMD approximation of order $\mathrm{n}$. From the (2) we define at $\omega=\omega_{0}$ the total DGD of the emulator as $\Delta \tau_{0}=|\vec{\Omega}|$ and its first derivative as $\Delta \tau_{0}^{\prime}$.

Exploiting the analytical relationship between the Jones matrix and the dispersion vector [5], the three components $\Omega_{1}, \Omega_{2}, \Omega_{3}$ of $\vec{\Omega}$ can be determined with respect to the Jones matrix coefficients $u_{1}, u_{2}$ from the equation: 


$$
\left\{\begin{array}{l}
\Omega_{1}=2 j\left(u_{1}^{*} u_{1}^{\prime}+u_{2}^{\prime} u_{2}^{*}\right) \\
\Omega_{2}=-\operatorname{Re}\left[2 j\left(u_{1}^{*} u_{2}^{\prime *}-u_{1}^{\prime *} u_{2}^{*}\right)\right] \\
\Omega_{3}=-\operatorname{Im}\left[2 j\left(u_{1}^{*} u_{2}^{\prime *}-u_{1}^{\prime *} u_{2}^{*}\right)\right]
\end{array}\right.
$$

In the following section we will study the statistics of $\Delta \tau_{0}, \Delta \tau_{0}^{\prime},\left|\vec{\Omega}_{0}^{\prime}\right|$ and $\left|\vec{\Omega}_{0}^{\prime \prime}\right|$ inserting $u_{1}, u_{2}$, calculated from equation (1) for the $N$-section emulator, into equation (3). The analytical solution of equation (3) is not easy for large $\mathrm{N}$ because of the complex form of the Jones matrix coefficients. If $\mathrm{N}=2$, the high-order PMD analytical model described in [5] can be obtained with an opportune rotation and under the conditions $\theta_{1}=0$ and $\delta \tau_{2}=-\delta \tau_{1} \cos \theta_{2}$. For $N=3$, equation (3) has been solved, giving at $\omega=\omega_{0}$ a dispersion vector of the form:

$$
\left\{\begin{array}{l}
\Omega_{01}=-\left[\delta \tau_{3}+\delta \tau_{2} \cos \left(2 \theta_{3}\right)+\delta \tau_{1} \cos \left(2 \theta_{3}\right) \cos \left(2 \theta_{2}\right)-\delta \tau_{1} \sin \left(2 \theta_{3}\right)\right. \\
\left.\cdot \sin \left(2 \theta_{2}\right) \cos \left(\delta \tau_{2} \omega_{0}\right)\right] \\
\Omega_{02}=-\sin \left(2 \theta_{3}\right)\left[\delta \tau_{2}+\delta \tau_{1} \cos \left(2 \theta_{2}\right)\right] \cos \left(\delta \tau_{3} \omega\right)-\delta \tau_{1} \sin \left(2 \theta_{2}\right) \cdot \\
{\left[\cos \left(2 \theta_{3}\right) \cos \left(\delta \tau_{2} \omega_{0}\right) \cdot \cos \left(\delta \tau_{3} \omega\right)-\sin \left(\delta \tau_{3} \omega_{0}\right) \sin \left(\delta \tau_{2} \omega_{0}\right)\right]} \\
\Omega_{0_{3}}=\sin \left(2 \theta_{3}\right)\left[\delta \tau_{2}+\delta \tau_{1} \cos \left(2 \theta_{2}\right)\right] \sin \left(\delta \tau_{3} \omega_{0}\right)+\delta \tau_{1} \sin \left(2 \theta_{2}\right) \cos \left(\delta \tau_{3} \omega_{0}\right) \\
\cdot\left[\sin \left(\delta \tau_{3} \omega_{0}\right) \cos \left(2 \theta_{3}\right)+\sin \left(\delta \tau_{2} \omega_{0}\right)\right]
\end{array}\right.
$$

In this case, the first rotator has been chosen with $\theta_{1}=0$. Fixing $\theta_{2}$ and varying $\theta_{3}$, or viceversa, equation (4) describes a complicated trajectory in the Stokes space. Note that, if the step angle is small, the number of possible trajectories increases and the emulated normalized dispersion vector tends to uniformly cover the Poincarè sphere, as expected [9]. At center-bandwidth the first derivative of the dispersion vector results to be 


$$
\left\{\begin{array}{l}
\Omega_{0_{1}}^{\prime}=f_{1}\left[\delta \tau_{1}, \delta \tau_{2}, \sin \left(2 \theta_{2}\right), \sin \left(2 \theta_{3}\right), \omega_{0}\right] \\
\Omega_{0_{2}}^{\prime}=f_{2}\left[\delta \tau_{1}, \delta \tau_{2}, \delta \tau_{3}, \sin \left(2 \theta_{2}\right), \sin \left(2 \theta_{3}\right), \cos \left(2 \theta_{2}\right), \cos \left(2 \theta_{3}\right), \omega_{0}\right] \\
\Omega_{0_{3}}^{\prime}=f_{3}\left[\delta \tau_{1}, \delta \tau_{2}, \delta \tau_{3}, \sin \left(2 \theta_{2}\right), \sin \left(2 \theta_{3}\right), \cos \left(2 \theta_{2}\right), \cos \left(2 \theta_{3}\right), \omega_{0}\right]
\end{array}\right.
$$

where $f_{i}[\cdot]$, with $\mathrm{i}=1,2,3$ are linear functions, not explicitly reported for simplicity. From the (4) and the (5), it can be observed that the 3 -section emulator lets to independently fix only two parameters among $\Delta \tau_{0}, \Delta \tau_{0}^{\prime}$ and $\left|\vec{\Omega}_{0}^{\prime}\right|$. Generalizing for $N$ sections, the first and high-order emulator parameters are functions of $\delta \tau_{i}$ and $\theta_{i}$, with $i=1, . ., N$ : consequently, the degree of freedom in the choice of the emulated parameters increases for increasing $\mathrm{N}$.

\section{RESULTS AND DISCUSSION}

Our study is focused on 3,5 and 8-section emulators. Being the number of sections physically limited, only low orders PMD parameters can be independently chosen. In the case of the 5 and 8 -section emulator, the first and second order parameters $\Delta \tau_{0}, \Delta \tau_{0}^{\prime}$ and $\left|\vec{\Omega}_{0}^{\prime}\right|$ are properly fixed, according to what explained in the previous section. In the 3-section case only $\Delta \tau_{0}$ and $\left|\vec{\Omega}_{0}^{\prime}\right|$ can be independently set at the emulator. As regards the third order PMD parameter $\left|\vec{\Omega}_{0}^{\prime \prime}\right|$, its values are calculated in the 8section case in correspondence of each fiber realization imposed to the emulator.

In Figg. 1, 2 and 3 the dark curves (blue line), representing the PDFs of $\Delta \tau_{0}, \Delta \tau_{0}$ and $\left|\vec{\Omega}_{0}^{\prime}\right|$ numerically obtained for $\left\langle\Delta \tau_{0}\right\rangle=7.5 \mathrm{ps}\left(0.3 \mathrm{~T}_{\mathrm{b}}\right.$ in a $40 \mathrm{~Gb} / \mathrm{s}$ transmission system, where $T_{b}$ is the bit time) with a 3-section $\left(\delta \tau_{\mathrm{i}}=6.5,4.1,2.4 \mathrm{ps}\right.$, for $\left.\mathrm{i}=1 . .3\right)$, a 5 -section $\left(\delta \tau_{\mathrm{i}}=3,2.4,3.2,4,5\right.$ ps for $\mathrm{i}=1 . .5)$ and a 8 -section emulator $\left(\delta \tau_{\mathrm{i}}=2.4,3,3.5,3.2,2.3,1.7,3.1,3.3 \mathrm{ps}\right.$ for $\mathrm{i}=1 . .8$ ), respectively, are compared to the analytical PDFs (red line). In all three cases the angles $\theta_{i}$ are varied on a wide range $\left(0-180^{\circ}\right)$ with different small steps (around $10^{\circ}$ ). The values of $\delta \tau_{i}$ are chosen to satisfy the relationship $\left\langle\Delta \tau_{0}{ }^{2}\right\rangle=\sum_{1}^{N}\left\langle\delta \tau_{i}^{2}\right\rangle$ [9]. To compute the numerical curves, we ask the emulator to satisfy 10000 different deterministic combinations of $\Delta \tau_{0}, \Delta \tau_{0}^{\prime}$ and $\left|\vec{\Omega}_{0}^{\prime}\right|$, taken from their analytical joint statistics. 
The 3-section emulator cannot describe the statistics neither of first nor of second PMD parameters. Increasing the number of sections, a good agreement between the simulated and the analytical curves is shown. We can say that the 8 -section case gives a very good statistical representation of these three PMD parameters.

Figg. 4 and 5 show the PDFs of $\Delta \tau_{0}, \Delta \tau_{0}^{\prime}$ and $\left|\vec{\Omega}_{0}^{\prime}\right|$ numerically obtained for $\left\langle\Delta \tau_{0}\right\rangle=13.3 \mathrm{ps}\left(0.5 \mathrm{~T}_{\mathrm{b}}\right.$ at $\left.40 \mathrm{~Gb} / \mathrm{s}\right)$ with a 5 -section $\left(\delta \tau_{\mathrm{i}}=\right.$ $6,8,5.5,7,5$ ps for $\mathrm{i}=1.5)$ and a 8 -section emulator, $\left(\delta \tau_{\mathrm{i}}=5,6,4,5.5,4.5\right.$, $3.5,6.5,4.8$ ps for $\mathrm{i}=1 . .8$ ), respectively. The same step for $\theta_{\mathrm{i}}$ and the same number of 'deterministic' realizations of Fig.1-3 are used. For these PMD conditions, the emulator with the lowest number of sections drastically underestimates the analytical curves. Whereas, the 8-section scheme shows a good approximation of the real PMD statistics up to second order, even on the tails of the PDFs.

By a comparison between Figg. 1, 2, 3 and Figg. 4, 5, it can be noticed that, to obtain a correct statistical characterization of a real PMD fiber, the number of delay sections needed increases as $\left\langle\Delta \tau_{0}\right\rangle$ gets larger. In fact, increasing the number of sections, the ranges for the emulated values of DGD and for the second order parameters become wider and, consequently, the possible number of fiber realizations increases. Real systems, generally, accept first order PMD distortions up to half bit time, hence, for a $40 \mathrm{~Gb} / \mathrm{s}$ system, is reasonable to consider DGDs lower than 13ps.

Fixing the number of sections and their delay, smaller step-angles increase the accuracy of the approximation given by the emulator. The reason can be found in the larger ensemble of values obtainable for the emulated PMD parameters, as shown in equations (4) and (5).

In Fig. 6 the PDF of the third order PMD parameter $\left|\vec{\Omega}_{0}^{\prime \prime}\right|$ is obtained for a 8 section emulator in the same case of Fig. 3. The light (red) dotted curve represents the PDF calculated with 100000 fiber realizations using the DRW numerical model, chosen as a faithful description of a real fiber affected by all order PMD effects. The discrepancies between the simulated curves and those calculated by the DRW model are containe in a little range. Moreover, the analytical relationship $\left\langle\left|\vec{\Omega}_{0}^{\prime \prime}\right|^{2}\right\rangle=\frac{4}{9}\left\langle\left|\vec{\Omega}_{0}\right|^{2}\right\rangle^{3}$ [8] is perfectly respected by the emulator results.

Finally Fig. 7 shows the same comparison in the case of $\left\langle\Delta \tau_{0}\right\rangle=13.3 \mathrm{ps}$; the simulated curve was obtained for a 8 -section emulator. The differences between the two curves increased but the approximation is still acceptable. 


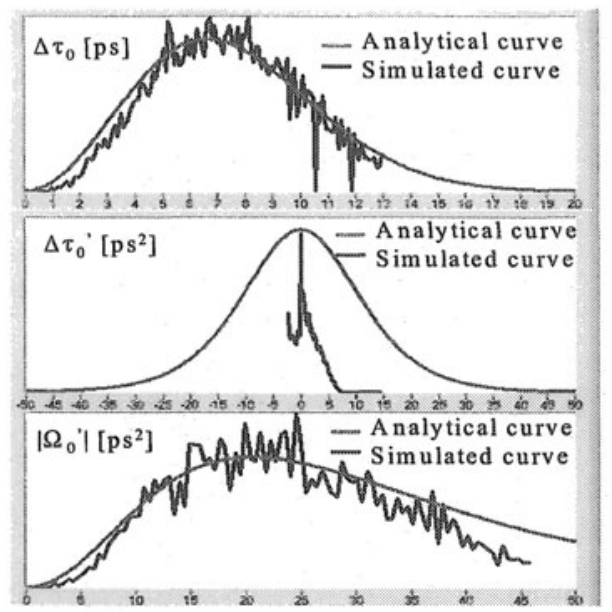

Figure 1. Analytical and simulated PDFs of $\Delta \tau_{0}, \Delta \tau_{0}^{\prime}$ and $\left|\vec{\Omega}_{0}^{\prime}\right|$, in the case of $\left\langle\Delta \tau_{0}>=7.5 \mathrm{ps}\right.$, for a 3-section emulator with $\delta \tau_{\mathrm{i}}=6.5,4.1,2.4 \mathrm{ps}, \mathrm{i}=1 . .3$.

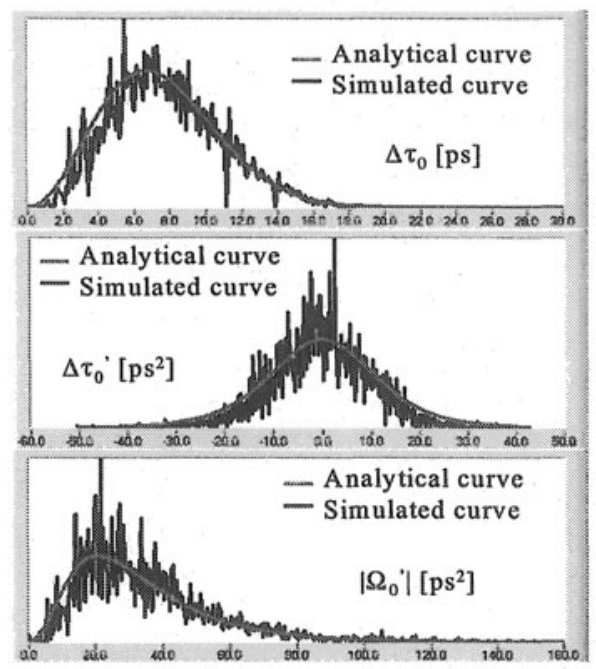

Figure 2. Analytical and simulated PDFs of $\Delta \tau_{0}, \Delta \tau_{0}^{\prime}$ and $\left|\vec{\Omega}_{0}^{\prime}\right|$, in the case of $\left\langle\Delta \tau_{0}>=7.5 \mathrm{ps}\right.$, for a 5-section emulator with $\delta \tau_{\mathrm{i}}=3,2.4,3.2,4,5 \mathrm{ps}, \mathrm{i}=1 . .5$. 


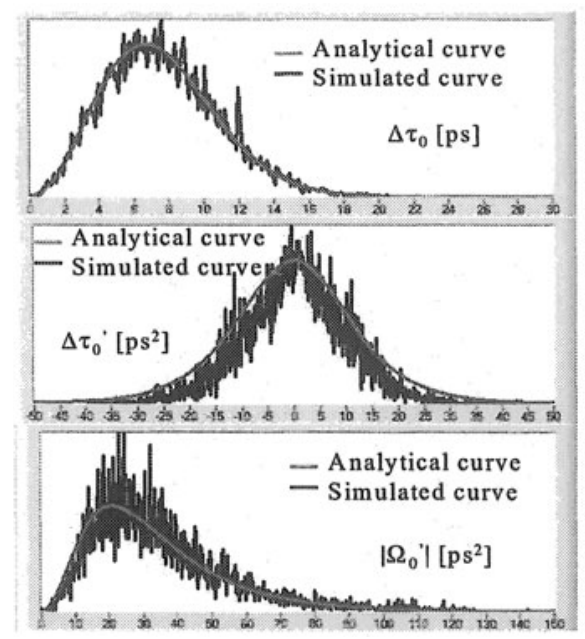

Figure 3. Analytical and simulated PDFs of $\Delta \tau_{0}, \Delta \tau_{0}^{\prime}$ and $\left|\vec{\Omega}_{0}^{\prime}\right|$, in the case of $<\Delta \tau_{0}>=7.5 \mathrm{ps}$, for a 8 -section emulator with $\delta \tau_{\mathrm{i}}=2.4,3,3.5,3.2,2.3,1.7,3.1,3.3 \mathrm{ps}$, $\mathrm{i}=1 . .8$.

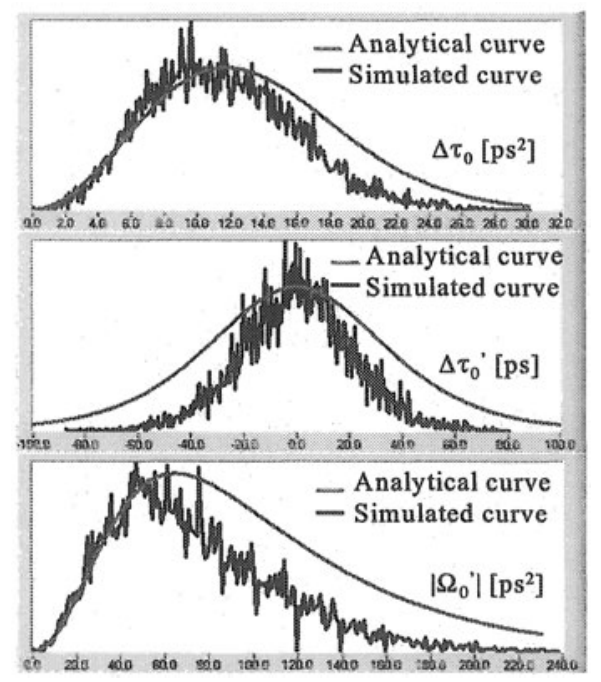

Figure 4. Analytical and simulated PDFs of $\Delta \tau_{0}, \Delta \tau_{0}^{\prime}$ and $\left|\vec{\Omega}_{0}^{\prime}\right|$, in the case of $<\Delta \tau_{0}>=13.3 \mathrm{ps}$, for a 5 -section emulator with $\delta \tau_{\mathrm{i}}=6,8,5.5,7,5 \mathrm{ps}, \mathrm{i}=1 . .5$. 


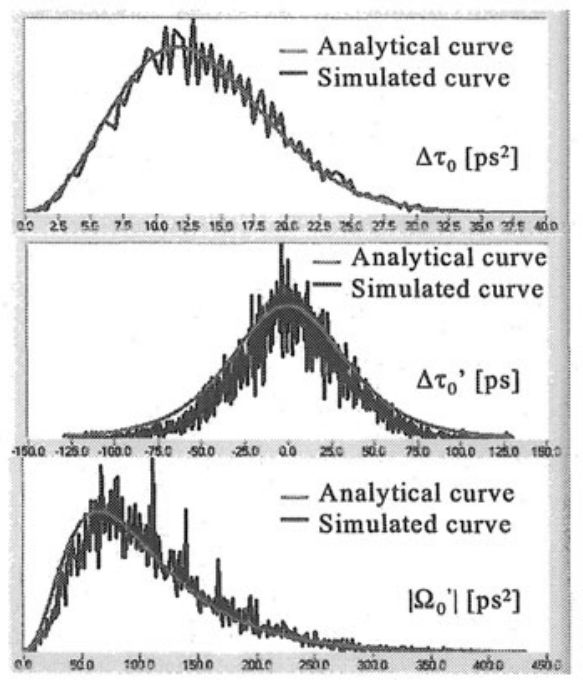

Figure 5. PDFs of $\Delta \tau_{0}, \Delta \tau_{0}^{\prime}$ and $\left|\vec{\Omega}_{0}^{\prime}\right|$, in the case of $\left\langle\Delta \tau_{0}\right\rangle=13.3$ ps, for a 8 section emulator with $\delta \tau_{\mathrm{i}}=2.4,3,3.5,3.2,2.3,1.7,3.1,3.3 \mathrm{ps}, \mathrm{i}=1 . .8$.

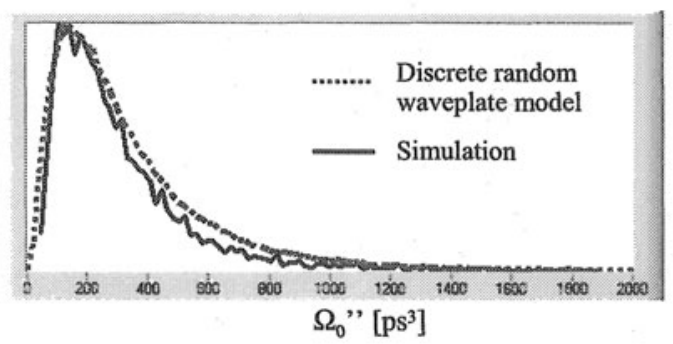

Figure 6. PDF of $\left|\vec{\Omega}_{0}^{\prime \prime}\right|\left[\mathrm{ps}^{3}\right]$ obtained by simulation (continuous) and by Discrete Random Waveplate model (dotted) in the case of $\left\langle\Delta \tau_{0}\right\rangle=7.5 \mathrm{ps}$, for a 8 -section emulator.

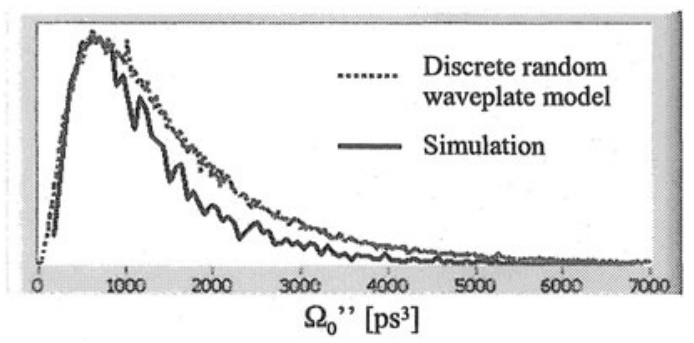

Figure 7. PDF of $\left|\vec{\Omega}_{0}^{\prime \prime}\right|\left[\mathrm{ps}^{3}\right]$ obtained by simulation (continuous) and by Discrete Random Waveplate model (dotted) in the case of $\left\langle\Delta \tau_{0}\right\rangle=13.3 \mathrm{ps}$, for a 8-section emulator. 


\section{CONCLUSIONS}

A deterministic emulator consisting of delay-sections with fixed DGD coupled by rotators is presented. The novel idea is creating a map of correspondence between the PMD emulator parameters and the rotation angles, that are varied according to an exhaustive scan. Imponing to the emulator the PMD statistics analytically known, the correspondent statistics of the coupling angles are easily determined. By this way it is possible to emulate the high-order PMD statistics of a real fiber with a reduced number of sections, even for rather high average DGDs.

\section{REFERENCES}

[1] J.N.Damask, "A programmable polarization-mode dispersion emulator for systematic testing of $10 \mathrm{~Gb} / \mathrm{s}$ PMD compensators," in Proc. OFC 2000, pp. 28-30, 2000.

[2] I.T.Lima, Jr, R.Khosravani, P.Ebrahimi, E.Ibragimov, A.E.Willner, and C.R.Menyuk, "Polarization mode dispersion emulator," in Proc. OFC 2000, pp. 31-33, 2000.

[3] O. Leminger and R. Leppla, "Statistical modelling of a higher order PMD emulator," in Proc. ECOC'01, pp. 344-345, 2001.

[4] G. Biondini, W. Kath, C. R. Menyuk, "Non-Maxwellian distribution of PMD emulators," in Proc. OFC 2001, ThA5, 2001.

[5] A. Orlandini and L. Vincetti "A simple and useful model for Jones matrix to evaluate higher order polarization mode dispersion effects," IEEE Photon. Technol. Lett.,vol. 13, no. 11, pp. 1176-1178, November 2001.

[6] G.Foschini and C.Poole, "Statistical theory of polarization dispersion in single mode fibers," IEEE J. Lightwave Technol., vol. 9, no. 11, pp. 1439-1456, November 1991.

[7] G. J. Foschini, L. E. Nelson, R. M. Jopson, and H. Kogelnik, "Probability densities of second order polarization mode dispersion including polarization dependent chromatic fiber dispersion," IEEE Photon. Technol. Lett., vol. 12, no. 3, pp. 293-295, March 2000.

[8] M. Sthaif, A. Mecozzi and J. A. Nagel, "Mean square magnitude of all orders of polarization mode dispersion and the relation with the bandwidth of the principal states," IEEE Photon. Technol. Lett., vol. 12, no. 1, pp. 53-55, January 2000.

[9] C.Poole and D.Favin, "Polarization mode-dispersion measurements based on transmission spectra through a polarizer," IEEE J. Lightwave Tech., vol.12, n.6, pp.917.929, June 1994. 